Original Article

\title{
Effects of proprioceptive sense-based Kinesio taping on walking imbalance
}

\author{
Young-Han Park, $\mathrm{PhD}^{1)}$, Jung-Ho Lee, $\mathrm{PhD}^{2)^{*}}$ \\ 1) Department of Physical Therapy, Korea National University of Transportation, Republic of Korea \\ 2) Department of Physical Therapy, Kyungdong University: Bongpo-ri, Toseong-myeon, Goseong-gun, \\ Gangwon-do, Republic of Korea
}

\begin{abstract}
Purpose] The aim of this study was to determine how application of Kinesio tape to the upper and lower limbs affects walking through stimulation of the proprioceptive sense. [Subjects and Methods] Twelve patients diagnosed with hemiplegia due to stroke were selected as the subjects of the study. To ascertain the effects of Kinesio taping on walking, all subjects performed a straight line walking test three times while barefoot. In terms of the actual taping application, elastic Kinesio tape was used on the hemiplegic side in all subjects. [Results] The results of testing showed a significant difference in the values between before and after taping. In terms of left and right deviation according to the site of the taping application, there were statistically significant differences among the groups. [Conclusion] In conclusion, application of Kinesio taping for central nerve injury was confirmed to be effective in reducing walking deviation.

Key words: Kinesio taping, Proprioception, Walking
\end{abstract}

(This article was submitted May 30, 2016, and was accepted Jul. 19, 2016)

\section{INTRODUCTION}

Stroke is described as a neurological disease that abruptly develops due to loss of brain function as a result of an abnormal decrease in the blood supply ${ }^{1}$. It is a primary cause of death and has a high incidence worldwide. Stroke is one of the most common neurological diseases in the world. In addition, it is the second most common neurological disease in developed countries and a major cause of impairment in adults. Although stroke patients may show normal walking patterns in some rare cases, most patients with stroke are incapable of walking normally due to problems with posture, muscle tone, and coordination of interactive movement. For approximately $80 \%$ of patients, a typical consequence of motor impairment after stroke is inability to control one side of the face and the upper and lower limbs ${ }^{2}$.

Balance in human beings is achieved by a process in which the central nervous system detects information entering the sensory organs and creates the necessary sensory integration, allowing the musculoskeletal system to produce an adequate response. Since the process the human body uses to maintain balance is complicated, poor posture that affects health is likely to develop once posture and balance are lost due to disease ${ }^{3)}$.

Posture control involves balance, motor ability, endurance, range of motion, sensory function, and muscle strength. The factors that hinder the maintenance of balance are diverse, and include loss of proprioceptive sense, neurological disease, joint contracture, and muscle weakening. The loss of proprioceptive sense affects learning and performance processes, and typically, it will induce inappropriate movement and imbalance. Generally, the proprioceptive sense maintains body balance through biofeedback mechanisms, and it is frequently used to correct motor performance during rehabilitation therapy ${ }^{4}$.

Kinesio taping, which is one of the methods that promotes the proprioceptive sense, limits excessive movement of the joints and improves proprioceptive feedback mechanisms to decrease muscle mobilization time ${ }^{5)}$. It also increases functional maintenance and flexibility of the joints, and addresses malfunctions of the musculoskeletal system ${ }^{6}$. Currently, Kinesio

*Corresponding author. Jung-Ho Lee (E-mail: 1jhcivapt@naver.com)

(C2016 The Society of Physical Therapy Science. Published by IPEC Inc.

This is an open-access article distributed under the terms of the Creative Commons Attribution Non-Commercial No Derivatives (by-nc-nd) License $<$ http://creativecommons.org/licenses/by-nc-nd/4.0/>. 
taping is being used as a conservative therapy method for relief of pain in the musculoskeletal system and for the recovery of muscle strength and function.

A number of studies have reported that training to improve proprioceptive sense using Kinesio taping improves body balance and walking ability. However, little research has been done on the effects of Kinesio taping on left and right deviation during walking. Hence, this study attempted to determine the effects of Kinesio taping on left and right deviation during walking in normal adults.

\section{SUBJECTS AND METHODS}

Among patients hospitalized at hospital C, 12 patients diagnosed with hemiplegia due to stroke were selected as the subjects of this study. All the subjects understood the purpose of the study and provided written informed consent prior to participation according to the ethical standards of the Declaration of Helsinki.

To ascertain the effects of Kinesio taping on walking in the subjects, all subjects performed a straight line walking test three times while barefoot using the three times according to the three methods described below. For the straight line walking test, a $10 \mathrm{~m}$ straight line was drawn on flat ground. The subjects first checked the start and end points of the line and then walked on the straight line while blindfolded. The error distance was measured three times, and the average value was used for the analysis.

In addition, an evaluation was conducted before Kinesio taping was applied, and an evaluation was conducted after Kinesio taping was applied. To minimize the effects of learning on the subjects due to repeated evaluation, 24 hours of rest was allowed between each taping method. The order of taping application was as follows: (1) taping was applied to the upper limb flexor (experimental group 1, EG1) (2) taping was applied to the lower limb flexor (experimental group 2, EG2); and (3) taping was applied to the lower and upper limb flexors (experimental group 3, EG3).

In terms of the actual taping application, elastic Kinesio tape (Kinesio Tex, Kinesio Co., Ltd., Tokyo, Japan) was used on the hemiplegic side of all subjects. For taping applied on the upper limb flexor, an I-shaped piece of Kinesio tape $2.5 \mathrm{~cm}$ wide was attached to the deltoid tuberosity of the lateral upper arm. Following extension of the shoulder joint, the end of the tape was attached to the clavicle. In the case of the brachioradialis, an I-shaped piece of Kinesio tape of $2.5 \mathrm{~cm}$ wide was extended as much as possible in a neutral wrist posture and attached from the styloid process of the hip bone to the lateral supracondylar ridge of the humerus.

For application of taping to the lower limb flexor, an I-shaped piece of Kinesio tape $2.5 \mathrm{~cm}$ wide was attached from the anterior superior iliac spine to the rectus femoris tendon. In the case of the anterior tibial muscle, an I-shaped piece of Kinesio tape of $2.5 \mathrm{~cm}$ wide was attached from the plantar surface of the first metatarsal bone to the peroneal muscle head covering the top of the foot.

The results of the evaluations before and after taping to examine left and right deviation during walking are presented as the mean \pm standard deviation. Statistical analysis was performed with IBM SPSS Statistics for Windows, Ver. 20.0. A paired t-test was used to check the differences in mean values before and after Kinesio taping application. One-way ANOVA was used to differences the difference between groups, and post hoc analysis was conducted using the LSD test. The significance level was set at $\mathrm{a}=0.5$.

\section{RESULTS}

The results of testing showed a significant difference in values between before and after the tape was applied in the case of EG3, with the difference being $33.64 \pm 6.85 \mathrm{~cm}(\mathrm{p}<0.05)$. There were also significant differences between before and after the tape was applied in the case of the upper limb (EG1) and the lower limb (EG2), with the differences being $18.65 \pm 4.59 \mathrm{~cm}$ and $23.15 \pm 6.48 \mathrm{~cm}$ (both $\mathrm{p}<0.05$ ), respectively (Table 1). In terms of left and right deviation according to site of the taping application, there were a statistically significant differences among the groups $(p<0.05)$. Comparison between EG3 and EG2 showed a statistically significant difference.

Table 1. Comparison of deviation between before and after taping

\begin{tabular}{lccc}
\hline & Before & After & Change $^{*}$ \\
\hline EG 1 $(\mathrm{cm})^{*}$ & $231.5 \pm 35.4$ & $213.6 \pm 32.4$ & $18.65 \pm 4.59$ \\
EG 2 $(\mathrm{cm})^{*}$ & $243.5 \pm 43.2$ & $220.4 \pm 28.6$ & $23.15 \pm 6.48$ \\
EG 3 $(\mathrm{cm})^{*}$ & $229.3 \pm 29.4$ & $195.3 \pm 40.2$ & $33.64 \pm 6.85$ \\
\hline
\end{tabular}

Values are presented as the mean $\pm \mathrm{SD} . * \mathrm{p}<0.05$. EG 1: upper limb taping group; $\mathrm{EG} 2$ : lower limb taping group; EG 3: upper and lower limb taping group 


\section{DISCUSSION}

Currently, taping is used to improve function in patients with a chronically unstable body and to prevent injury in those with a healthy body. The mechanism of taping therapy originates from the cutaneous fusimotor reflex theory. Stimulation of the skin through the physical stimulation of tape, specifically by means of touch, pressure, and vibration, induces continuous contraction of the muscle under the taped skin through gamma reflexes. Since it resolves muscle tone via the relaxation that instinctively develops after muscle contraction, taping results in pain relief and muscle relaxation ${ }^{7}$.

Stroke patients who support approximately 30 to $40 \%$ of their body weight on the paralyzed side experience diverse problems, including instability of left and right balance, instability of postural control, and a decrease in posture alignment and balance due to diminished motor ability. Moreover, foot drop, which is caused by difficulty with ankle joint dorsiflexion, is one of the most frequent complications occurring in stroke patients. Abnormal coordination of the lower limb flexor and extensor occurs due to compensation on the unaffected side, proprioceptive sense injury, and ankle joint stiffness ${ }^{8)}$.

Most patients experience impairment in walking ability after stroke, and $40 \%$ of patients need help to recover their walking ability. Although $60 \%$ resume walking after stroke, they are still subject to restrictions in their ability to move independently outside the home. Despite efforts aimed at rehabilitation, approximately $35 \%$ of stroke patients with lower limb paralysis do not fully recover their walking ability, while approximately $25 \%$ of stroke patients cannot walk without the aid of others before being discharged from the hospital ${ }^{9}$. In most cases, the walking function of patients is reported to be affected by neurological impairment, and this is apparently what induces problems with asymmetrical gait. Hence, recovery of walking function following stroke should be one of the main goals of rehabilitation ${ }^{10)}$.

In this study, EG3 taping, EG1 taping, and EG2 taping, used to stimulate the proprioceptive sense, showed statistically significant differences in the results of a straight line walking test. Although not all joints were measured due to difficulties in terms of research implementation, measurement of joints on the hemiplegic side indicated statistically significant results. Thus, it can be concluded that applying tape to the skin increases cutaneous sensation; in other words, taping provides a strong proprioceptive cue through its contact with the skin, and the resulting cutaneous sensory information can decrease a stroke patient's left and right deviation when walking.

This study has some limitations, in that it did not consider physiological and psychological factors relating to the subjects. Moreover, no three-dimensional gait analysis was conducted after taping, and the study results also have limitations in terms of generalization due to the lack of a control group. However, using the results of this study as fundamental data, we hope that future studies will make further progress by evaluating the effects of proprioceptive stimulation using taping on the walking ability of stroke patients through three-dimensional motion analysis or by comparing the above results with those of other therapy methods.

\section{ACKNOWLEDGEMENT}

This was supported by Korea National University of Transportation in 2016.

\section{REFERENCES}

1) Jørgensen HS, Nakayama H, Raaschou HO, et al.: Recovery of walking function in stroke patients: the Copenhagen Stroke Study. Arch Phys Med Rehabil, 1995, 76: 27-32. [Medline] [CrossRef]

2) Perry J, Garrett M, Gronley JK, et al.: Classification of walking handicap in the stroke population. Stroke, 1995, 26: 982-989. [Medline] [CrossRef]

3) Balasubramanian CK, Bowden MG, Neptune RR, et al.: Relationship between step length asymmetry and walking performance in subjects with chronic hemiparesis. Arch Phys Med Rehabil, 2007, 88: 43-49. [Medline] [CrossRef]

4) Horstman AM, Beltman MJ, Gerrits KH, et al.: Intrinsic muscle strength and voluntary activation of both lower limbs and functional performance after stroke. Clin Physiol Funct Imaging, 2008, 28: 251-261. [Medline] [CrossRef]

5) Jaraczewska E, Long C: Kinesio taping in stroke: improving functional use of the upper extremity in hemiplegia. Top Stroke Rehabil, 2006, 13: 31-42. [Medline] [CrossRef]

6) Shim S, Jung J: Effects of bilateral training on motor function, amount of activity and activity intensity measured with an accelerometer of patients with stroke. J Phys Ther Sci, 2015, 27: 751-754. [Medline] [CrossRef]

7) Zanca GG, Grüninger B, Mattiello SM: Effects of Kinesio taping on scapular kinematics of overhead athletes following muscle fatigue. J Electromyogr Kinesiol, 2016, 29: 113-120. [Medline] [CrossRef]

8) Jeong YG, Koo JW: The effects of treadmill walking combined with obstacle-crossing on walking ability in ambulatory patients after stroke: a pilot randomized controlled trial. Top Stroke Rehabil, 2016, 20: 1-7. [Medline] [CrossRef]

9) Park KT, Kim HJ: Effect of the a circuit training program using obstacles on the walking and balance abilities of stroke patients. J Phys Ther Sci, 2016, 28: 1194-1198. [Medline] [CrossRef]

10) Polese JC, Servio TC, Chaves GS, et al.: Relationships between self-reported and performance-based measures of functional capacity in individuals with chronic stroke. J Phys Ther Sci, 2016, 28: 1208-1212. [Medline] [CrossRef] 